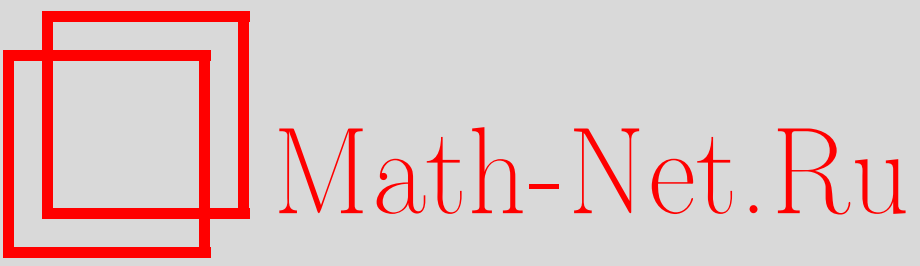

В. П. Макаров, О потенциале Брейта, ТМФ, 2006, том 146, номер 3, 443-446

DOI: https://doi.org/10.4213/tmf2046

Использование Общероссийского математического портала Math-Net.Ru подразумевает, что вы прочитали и согласны с пользовательским соглашением

http://www.mathnet.ru/rus/agreement

Параметры загрузки:

IP : 54.162 .85 .209

26 апреля 2023 г., 04:03:44

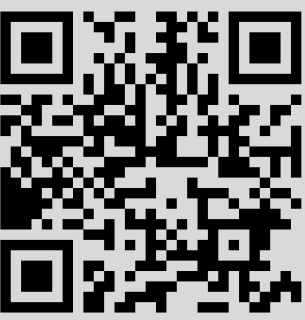


ТЕОРЕТИЧЕСКАЯ

И МАТЕМАТИЧЕСКАЯ

ФИЗИКА

Том 146, № 3

март, 2006

(C) 2006 г.

В. П. Макаров*

\section{О ПОТЕНЦИАЛЕ БРЕЙТА}

Показано, что критика известного результата для спин-спиновой части потенциала Брейта (см. статью Л. С. Кузьменкова, С. Г. Максимова, В. В. Федосеева) ошибочна. Доказано, что формула для потенциала Брейта, приведенная в книге В. Б. Берестецкого, Е. М. Лифшица, Л. П. Питаевского, правильна.

Ключевые слова: частицы со спином $1 / 2$, спин-спиновое взаимодействие, потенциал Брейта.

Оператор $\widehat{U}$ энергии взаимодействия между двумя частицами со спином $1 / 2$, записанный с точностью до членов порядка $(1 / c)^{2}$, носит название потенциала Брейта [1]. Берестецкий и Ландау [2] получили правильное выражение для спиновой части оператора $\widehat{U}$ :

$$
\widehat{U}_{\text {spin }}(\vec{r})=\frac{e^{2} \hbar^{2}}{4 m_{1} m_{2} c^{2}}\left\{\left[\frac{\left(\hat{\vec{\sigma}}_{1} \hat{\vec{\sigma}}_{2}\right) r^{2}-3\left(\hat{\vec{\sigma}}_{1} \vec{r}\right)\left(\hat{\vec{\sigma}}_{2} \vec{r}\right)}{r^{5}}\right]^{\prime}-\frac{8 \pi}{3} \hat{\vec{\sigma}}_{1} \hat{\vec{\sigma}}_{2} \delta(\vec{r})\right\},
$$

где $m_{i} \quad(i=1,2)$ - массы частиц, $e_{1}=e_{2}=e$ - заряд каждой частицы, $r$ - расстояние между частицами и $\hat{\vec{\sigma}}_{i}$ - спиновые матрицы Паули. Во всех работах, опубликованных до этого, отсутствует второе слагаемое в $\widehat{U}_{\text {spin }}(\vec{r})$ (обзор работ, в которых исследуется потенциал Брейта, дан Бете и Солпитером в книге [3], с. 269-289). Обозначение [... ]' в (1) соответствует замечанию, имеющемуся в [2]: “в интегралах от $[\ldots]^{\prime}$ можно не обращать внимания на их кажущуюся расходимость и, например, проводить первым интегрирование по углам". То же самое по существу замечание выражено в [3] следующим образом: “если член типа [...]' встречается в любом интеграле по координатному пространству, его заменяют нулем при $r<\varepsilon$, берут интеграл и переходят к пределу при $\varepsilon \rightarrow 0$ ".

Как отмечается в [3], вычисление оператора энергии взаимодействия удобнее проводить сначала в импульсном представлении (см. также с. 378-381 в [4]):

$$
\widehat{U}_{\text {spin }}(\vec{q})=\frac{\pi e^{2} \hbar^{2}}{m_{1} m_{2} c^{2} q^{2}}\left\{\left(\hat{\vec{\sigma}}_{1} \vec{q}\right)\left(\hat{\vec{\sigma}}_{2} \vec{q}\right)-q^{2}\left(\hat{\vec{\sigma}}_{1} \hat{\vec{\sigma}}_{2}\right)\right\},
$$

*Институт общей физики им. А.М. Прохорова РАН, Москва, Россия. Е-mail: asam@ran.gpi.ru 
где $\hbar \vec{q}=\vec{p}_{1}^{\prime}-\vec{p}_{1}=\vec{p}_{2}-\vec{p}_{2}^{\prime}-$ импульс, переданный при рассеянии первой частицей второй. Фурье-преобразование оператора (2) приводит к результату БерестецкогоЛандау (1):

$$
\widehat{U}_{\text {spin }}(\vec{r})=\frac{1}{(2 \pi)^{3}} \int d \vec{q} \widehat{U}_{\text {spin }}(\vec{q}) e^{i \vec{q} \vec{r}} .
$$

В 2001 г. была опубликована работа [5], результаты которой (в той ее части, которая касается потенциала Брейта) состоят в следующем: формула (2) для потенциала в импульсном представлении правильная, но формула для спин-спинового потенциала в координатном представлении (1) не корректна. Мы покажем сначала, что последнее утверждение ошибочно, а потом обсудим аргументацию авторов работы [5]. Для этого проведем Фурье-преобразование потенциала (1), который в [5] считается неправильным, и покажем, что в результате получается формула (2), которая и в [5] считается правильной. В интеграле

$$
\widehat{\widetilde{U}}(\vec{q}) \equiv \int d \vec{r} \widehat{U}_{\text {spin }}(\vec{r}) e^{-\vec{q} \vec{r}},
$$

где $\widehat{U}_{\text {spin }}(\vec{r})$ задается формулой $(1)$, вводим сферические координаты $r, \theta, \varphi$ с осью $z$ вдоль вектора $\vec{q}$. После интегрирования по $\varphi$ имеем

$$
\begin{aligned}
\widehat{\widetilde{U}}_{\text {spin }}(\vec{q})= & \frac{\pi e^{2} \hbar^{2}}{m_{1} m_{2} c^{2} q^{2}}\left\{q^{2}\left(\hat{\vec{\sigma}}_{1} \hat{\vec{\sigma}}_{2}\right)-3\left(\hat{\vec{\sigma}}_{1} \vec{q}\right)\left(\hat{\vec{\sigma}}_{2} \vec{q}\right)\right\} \times \\
& \times \int_{0}^{\infty} \frac{d r}{r} \int_{0}^{\pi} d \theta \sin \theta\left(3 \cos ^{2} \theta-1\right) e^{-i q r \cos \theta}-\frac{2 \pi e^{2} \hbar^{2}}{3 m_{1} m_{2} c^{2}}\left(\hat{\vec{\sigma}}_{1} \hat{\vec{\sigma}}_{2}\right) .
\end{aligned}
$$

После очевидной замены переменной $\eta=\cos \theta$ и элементарного интегрирования по $\eta$ получим

$$
\begin{aligned}
\widehat{\widetilde{U}}(\vec{q})= & \frac{\pi e^{2} \hbar^{2}}{m_{1} m_{2} c^{2} q^{2}}\left\{q^{2}\left(\hat{\vec{\sigma}}_{1} \hat{\vec{\sigma}}_{2}\right)-3\left(\hat{\vec{\sigma}}_{1} \vec{q}\right)\left(\hat{\vec{\sigma}}_{2} \vec{q}\right)\right\} \times \\
& \times \int_{0}^{\infty} \frac{d \rho}{\rho^{2}}\left(\sin \rho+\frac{3 \cos \rho}{\rho}-\frac{3 \sin \rho}{\rho^{2}}\right)-\frac{2 \pi e^{2} \hbar^{2}}{3 m_{1} m_{2} c^{2}}\left(\hat{\vec{\sigma}}_{1} \hat{\vec{\sigma}}_{2}\right) .
\end{aligned}
$$

Легко проверить, что интеграл в (6) сходится. Поэтому нижний предел можно заменить на некоторую конечную величину $\varepsilon>0$, вычислить интеграл от каждого из трех слагаемых по отдельности, а затем перейти к пределу $\varepsilon \rightarrow 0$. Вычислим сначала интеграл, содержащий $\rho^{-4}$, затем интеграл, содержащий $\rho^{-3}$, и в результате получим, что интеграл в $(6)$ равен $(-1 / 3)$, а $\widehat{\widetilde{U}}(\vec{q})=\widehat{U}_{\text {spin }}(\vec{q})$, как и должно быть, если формула (1) правильная.

Теперь перейдем к обсуждению того, как авторы [5] доказывают свое утверждение о некорректности потенциала (1). Из (2) и (3) следует формула (14) из [5] ${ }^{1)}$, совпадающая с соответствующей формулой в [3]-[4]:

$$
\widehat{U}_{\text {spin }}(\vec{r})=-\frac{e^{2} \hbar^{2}}{4 m_{1} m_{2} c^{2}} \hat{\sigma}_{1}^{\alpha} \hat{\sigma}_{2}^{\beta}\left(\frac{\partial^{2}}{\partial x^{\alpha} \partial x^{\beta}} \frac{1}{r}+4 \pi \delta^{\alpha \beta} \delta(\vec{r})\right) .
$$

\footnotetext{
${ }^{1)}$ В обозначениях мы следуем работе [5].
} 
Следующий шаг, который согласно [5] нужно сделать, чтобы из (7) получить $\widehat{U}_{\text {spin }}(\vec{r})$ в форме $(1)$, состоит в использовании равенства

$$
-\frac{\partial^{2}}{\partial x^{\alpha} \partial x^{\beta}} \frac{1}{r}=\frac{\delta^{\alpha \beta}}{r^{3}}-\frac{3 x^{\alpha} x^{\beta}}{r^{5}}+\frac{4 \pi}{3} \delta^{\alpha \beta} \delta(\vec{r}) .
$$

Авторы [5] правы в том, что равенство (8) некорректно. Авторы, однако, ошибаются, полагая, что это равенство необходимо для перехода от (7) к потенциалу в форме (1). Дело в том, что решение любой физической задачи содержит оператор $\widehat{U}_{\text {spin }}(\vec{r})$, как и полный оператор энергии взаимодействия $\widehat{U}$, только в виде соответствующих интегралов: сами операторы получены в [2]-[5] при расчете амплитуды рассеяния двух частиц со спином $1 / 2$.

Матричный элемент оператора (7), соответствующий переходу частиц из некоторого начального состояния в некоторое конечное состояние, после интегрирования по координатам центра масс содержит интегралы вида

$$
I_{\alpha \beta}=\int d \vec{r} f(\vec{r}) \frac{\partial^{2}}{\partial x^{\alpha} \partial x^{\beta}} \frac{1}{r}=\int d \vec{r} \frac{1}{r} \frac{\partial^{2} f(\vec{r})}{\partial x^{\alpha} \partial x^{\beta}} ;
$$

здесь $f(\vec{r})$ - произведение волновой функции относительного движения частиц в начальном состоянии и функции, комплексно сопряженной волновой функции относительного движения частиц в конечном состоянии; мы полагаем, разумеется, что интегралы существуют (сходятся). Для перехода от оператора (7) к оператору (1) достаточно выполнения следующего равенства:

$$
I_{\alpha \beta}=\int d \vec{r}\left[\frac{3 x^{\alpha} x^{\beta}-\delta^{\alpha \beta} r^{2}}{r^{5}}\right]^{\prime} f(\vec{r})-\frac{4 \pi}{3} \delta^{\alpha \beta} f(0)
$$

или, поскольку функция $f(\vec{r})$ произвольна, равенства (ср. с (8))

$$
-\frac{\partial^{2}}{\partial x^{\alpha} \partial x^{\beta}} \frac{1}{r}=\left[\frac{\delta^{\alpha \beta}}{r^{3}}-\frac{3 x^{\alpha} x^{\beta}}{r^{5}}\right]^{\prime}+\frac{4 \pi}{3} \delta^{\alpha \beta} \delta(\vec{r}),
$$

которое, однако, можно и нужно понимать только в смысле (10). Из (7) и (11) следует формула (1).

Что касается формулы (11), то она представляет собой частный случай формулы, которая получается в теории обобщенных функций, определенных в $n$-мерном вещественном пространстве $\mathbb{R}_{n}$, состоящем из точек $x=\left(x^{1}, x^{2}, \ldots, x^{\alpha}, \ldots, x^{n}\right)$, (см. [6], $\S 11$, задачи 14 и 13$)$ :

$$
\frac{\partial^{2}}{\partial x^{\alpha} \partial x^{\beta}} \frac{1}{r^{n-2}}=(n-2)\left(\frac{n x^{\alpha} x^{\beta}-r^{2} \delta^{\alpha \beta}}{r^{n+2}}\right)^{\prime}-\frac{n-2}{n} \Omega_{n} \delta^{\alpha \beta} \delta(x),
$$

где $r^{2}=\left(x^{\alpha}\right)^{2}, \delta(x)=\delta\left(x^{1}\right) \ldots \delta\left(x^{n}\right)$ и $\Omega_{n}$ - площадь сферы радиуса $r=1$ в $\mathbb{R}_{n}$. Для случая трехмерного пространства $(n=3)$ формула (12) переходит в формулу (11). 
Благодарности. Автор благодарен рецензенту, указавшему на возможность приведенного здесь обоснования формулы (11).

\section{Список литературы}

[1] G. Breit. Phys. Rev. 1929. V. 34. P. 553; 1932. V. 39. P. 616.

[2] В.Б. Берестецкий, Л.Д. Ландау. ЖЭТФ. 1949. Т. 19. С. 673.

[3] Г. Бете, Э. Солпитер. Квантовая механика атомов с одним и двумя электронами. М.: ГИФМЛ, 1960.

[4] В.Б. Берестецкий, Е.М. Лифиии, Л.П. Питаевский. Квантовая электродинамика. М.: Наука, 1980.

[5] Л. С. Кузъменков, С.Г. Максимов, В.В. Федосеев. ТМФ. 2001. Т. 126. С. 136.

[6] Г. Е. Шилов. Математический анализ. Второй специальный курс. М.: Наука, 1965.

Поступила в редакцию 17.III.2005 г., после доработки 6.IX.2005 г. 